\title{
Escherichia coli 0103 outbreak associated with minced celery among hospitalized individuals in Victoria, British Columbia, 2021
}

\author{
Courtney Smith ${ }^{1 \star}$, Allison Griffiths², Sandra Allison², Dee Hoyano², Linda Hoang ${ }^{3}$
}

\begin{abstract}
Background: In April 2021, a Shiga toxin-producing Escherichia coli (E. coli) (STEC) O103 outbreak was identified among patients at two hospitals in Victoria, British Columbia (BC). The objective of this study is to describe this outbreak investigation and identify issues of food safety for high-risk products prepared for vulnerable populations.

Methods: Confirmed cases of E. coli $\mathrm{O} 103$ were reported to the Island Health communicable disease unit. The provincial public health laboratory conducted whole genome sequencing on confirmed case isolates, as per routine practice for STEC in BC. Exposure information was obtained through case interviews and review of hospital menus. Federal and local public health authorities conducted an inspection of the processing plant for the suspect source.
\end{abstract}

Results: Six confirmed cases of $E$. coli 0103 were identified, all related by whole genome sequencing. The majority of cases were female $(67 \%)$ and the median age was 61 years (range 24-87 years). All confirmed cases were inpatients or outpatients at two hospitals and were exposed to raw minced celery within prepared sandwiches provided by hospital food services. A local processor supplied the minced celery exclusively to the two hospitals. Testing of product at the processor was infrequent, and chlorine rinse occurred before mincing. The spread of residual $E$. coli contamination through the mincing process, in addition to temperature abuse at the hospitals, are thought to have contributed to this outbreak.

Conclusion: Raw vegetables, such as celery, are a potential source of STEC and present a risk to vulnerable populations. Recommendations from this outbreak include more frequent testing at the processor, a review of the chlorination and mincing process and a review of hospital food services practices to mitigate temperature abuse.
This work is licensed under a Creative Commons Attribution 4.0 Internationa License.

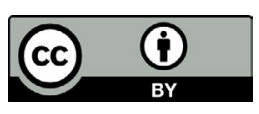

Affiliations

${ }_{1}$ Public Health Agency of Canada, Victoria, BC

${ }^{2}$ Island Health, Victoria, BC

${ }^{3}$ British Columbia Centres for Disease Control, Vancouver, BC

*Correspondence:

courtney.r.smith@phac-aspc.gc.ca

Suggested citation: Smith CR, Griffiths A, Allison S, Hoyano D, Hoang L. Escherichia coli O103 outbreak associated with minced celery among hospitalized individuals in Victoria, British Columbia, 2021. Can Commun Dis Rep 2022;48(1):47-50. https://doi.org/10.14745/ccdr.v48i01a07

Keywords: E. coli infection, foodborne, disease outbreak, celery

\section{Introduction}

Foodborne illness caused by Escherichia coli (E. coli) often occurs through the consumption of contaminated food items such as fresh produce, meat and cheese products, and may result in symptoms including watery diarrhea, hemorrhagic colitis and hemolytic uremic syndrome $(1,2)$. Pathogenic Shiga toxinproducing E. coli (STEC) are amongst the top 10 most common causes of foodborne illness in Canada (3). Although E. coli O157 remains the more common STEC, incidence rates of non-O157 STEC infections, including E. coli O103, have increased over time. The main factor contributing to this increase is an advancement in diagnostic testing (4).
E. coli $\mathrm{O} 103$ outbreaks have previously been linked to clover sprouts, bison meat, ground beef, cured mutton sausages, raw cow milk and fermented sausages (5-8). Although celery has been reported as a vehicle for Listeria monocytogenes, norovirus and E. coli O157:H7 (9-11), there have been no outbreaks of non-O157 E. coli associated with celery reported in the literature to date.

In April 2021 a Shiga toxin-producing E. coli O103 outbreak was identified among inpatients and outpatients at two hospitals in Victoria, British Columbia (BC), after an unusual increase in E. coli 
activity triggered an investigation by local public health officials. The objective of this article is to describe the first outbreak of non-O157 E. coli associated with celery in Canada and to identify issues of food safety for high-risk products prepared for vulnerable populations, in order to reduce the likelihood of these outbreaks in the future.

\section{Methods}

All STEC cases are reportable to public health within BC. Local hospital and community laboratories in Victoria screen enteric samples for Stx genes (12). If positive, the local regional laboratory in Victoria tests samples for STEC isolation in culture, and these isolates are forwarded to $B C$ Centre for Disease Control Public Health Laboratory for serotyping and whole genome sequencing (WGS). All STEC received at, or recovered by, the Public Health Laboratory are routinely serotyped using a multiplex polymerase chain reaction targeting the most common serotypes in BC: O26; O45; O111; O103; O121; and O145. All STEC isolates routinely undergo whole genome multi-locus sequence typing (wgMLST). The wgMLST schema for $E$. coli compared 17,380 loci in the E. coli genome according to standardized procedures used by PulseNet Canada. As per PulseNet Canada, E. coli isolates were considered genetically related if they are within 10 allele differences.

An unusual increase in E. coli $\mathrm{O} 103$ cases was detected in April 2021 in the Victoria area, which triggered an investigation to identify the source of the illness. The outbreak investigation took place between April 16, 2021, and May 10, 2021. A confirmed case was defined as a resident of or visitor to the Island Health region with laboratory confirmation of E. coli $\mathrm{O} 103$ and symptom onset or collection date on or after March 15, 2021. Cases were interviewed by a single interviewer with $B C$ 's routine $E$. coli questionnaire. The interviews collected information on travel, animal exposures and select high-risk foods associated with previous $E$. coli outbreaks, including beef, leafy greens and unpasteurized dairy. Exposure information was collected for the 10-day period prior to the episode date (earliest of symptom onset or specimen collection date), reflecting the incubation period of $E$. coli. For those admitted to hospital during their incubation period, hospital menus were also reviewed for the 10-day period prior to their episode date.

Local investigators inspected the kitchen of Hospital A, where the majority of cases were inpatients or outpatients. The inspectors examined cooler temperatures and logs, dishwasher temperatures, sanitizing processes, dating of product and food handling practices for any deficiencies or potential for cross contamination. Inspectors also inquired about ill food handlers. Records were reviewed to determine the suppliers of various products.

Local and federal investigators inspected the processing facility of the suspect source of the outbreak-Processor A. Inspectors collected supply records and investigated processes to determine potential sources of contamination and potential deficiencies in food safety.

The outbreak was declared over when the maximum incubation period ( 10 days) plus $90^{\text {th }}$ percentile reporting delay had passed since the most recent episode date of a confirmed case.

\section{Results}

Six confirmed cases were identified throughout the course of the investigation. Episode dates ranged from March 20 to April 9, 2021 (Figure 1). The majority of cases were female $(n=4 / 6 ; 67 \%)$ and the median age was 61 years (age range $24-87$ years). One death was reported ( $n=1 / 6 ; 17 \%)$, although E. coli infection was not the cause of death. All cases had been admitted to, or visited, two Victoria-area hospitals during their exposure period. Of the six confirmed cases, four were admitted to Hospital A, one was admitted to Hospital B, and one case was not admitted to hospital, but visited the emergency room of Hospital A during the exposure period (Figure 2). For those cases with onset dates available, the median reporting delay was 19 days (range 18-23 days).

\section{Figure 1: Confirmed cases of Escherichia coli 0103 infection by episode date (earliest of symptom onset or specimen collection date), March-April 2021}

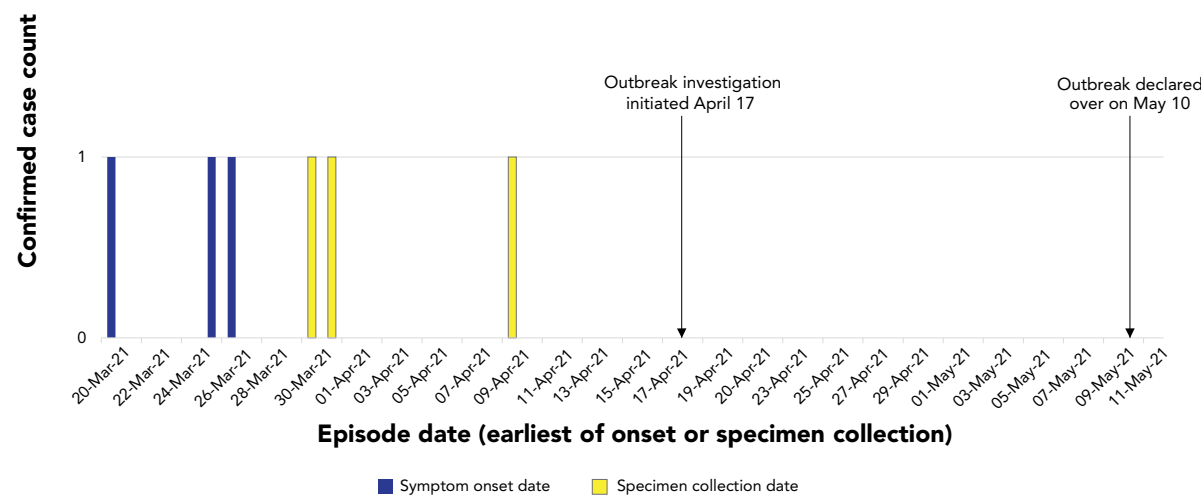


Figure 2: Gantt chart of confirmed cases indicating exposure period, episode date (earliest of symptom onset or specimen collection date), and dates in hospital

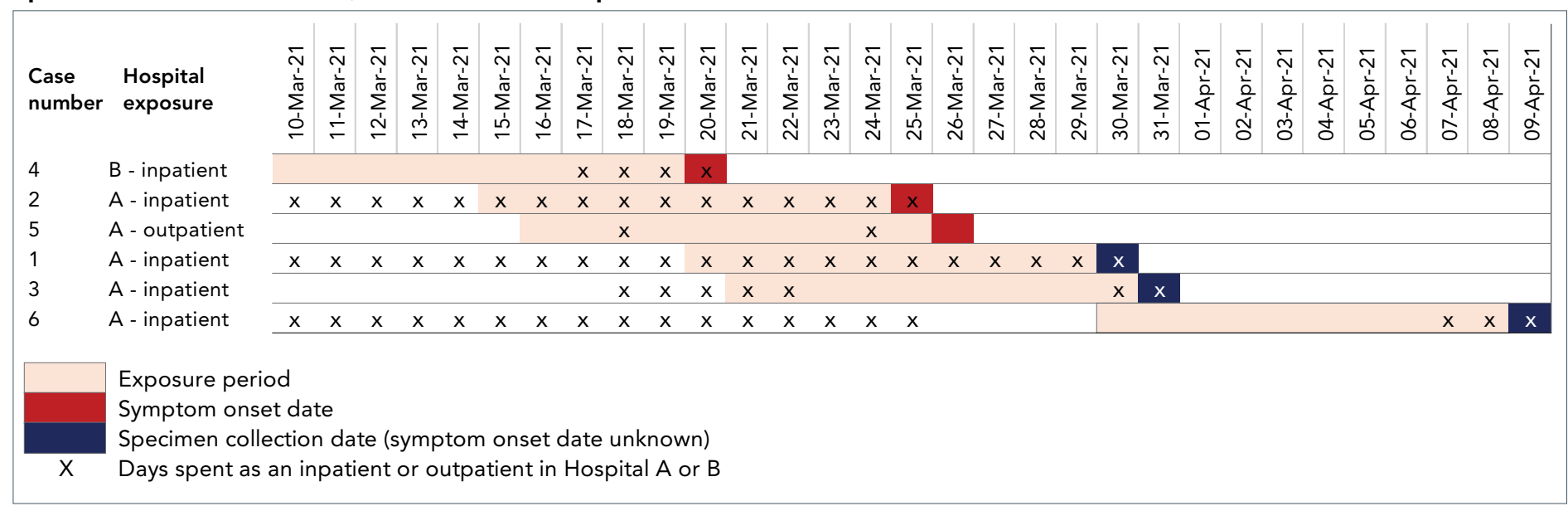

All confirmed cases from both Hospital A and Hospital B were considered highly related to each other by wgMLST within zero to four allele differences (Figure 3), and distinct from historic cases of $E$. coli 0103 . There were no related cases identified within the same timeframe of this outbreak nationally, or within the United States.

Figure 3: Phylogenic tree of Escherichia coli 0103

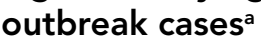

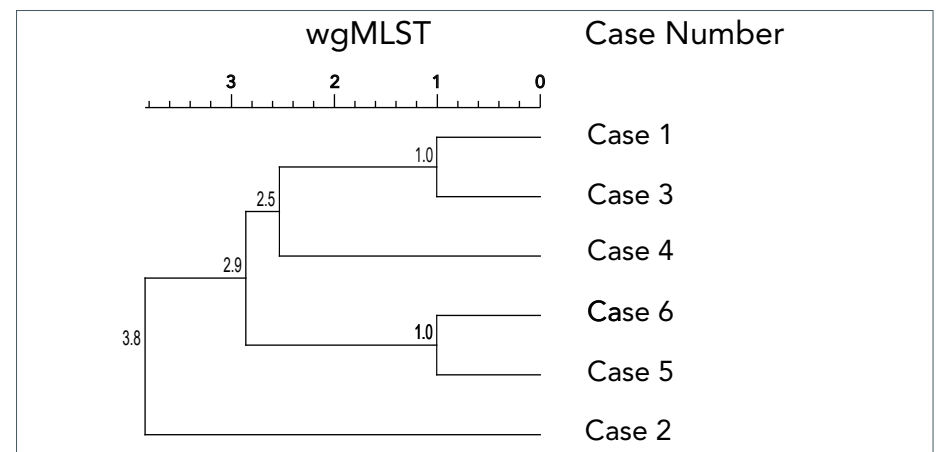

Abbreviation: wgMLST, whole genome multi-locus sequence typing

a Comparisons were generated using Bionumerics v 7.6.2. The wgMLST allele differences indicated at the nodes were calculated using unweighted pair group method with arithmetic mean (UPGMA) and therefore, are not whole numbers. To determine the nearest whole number value, round up if the digit following the decimal is five or greater; round down if the digit following the decimal is less than five

Three cases were interviewed, while the remaining three could not be interviewed as they were either deceased $(n=1)$ or medically impaired $(n=2)$. From the interview data, the only exposures reported by at least two of three confirmed cases were ground beef $(n=2 / 3)$, cheese $(n=3 / 3)$, cold cuts $(n=2 / 3)$, lettuce $(n=2 / 3)$ and tuna sandwiches $(n=3 / 3)$. No commonalities in restaurants or grocery stores were identified. The case that visited the emergency room of Hospital A reported eating only a tuna sandwich during their visit, given to them by a healthcare worker. The tuna sandwich was prepared by Hospital A food services and came from the same source as the inpatient food.
Hospital menus were reviewed for the five cases that were admitted to hospital. All five cases had exposure to prepared sandwiches during their hospital stay including tuna $(n=4 / 5)$, egg $(n=2 / 5)$, chicken salad $(n=4 / 5)$, turkey $(n=1 / 5)$ and roast beef $(n=4 / 5)$ sandwiches. The only common ingredient across all sandwiches was minced celery, and minced celery within sandwiches was the only exposure reported by $n=6 / 6$ cases. Inspection of the kitchen of Hospital A, where the majority of cases were exposed to minced celery as either inpatients or outpatients, revealed no food safety or cross-contamination concerns. There were no reported illnesses among food handling staff. The inspection did reveal concerns regarding temperature abuse, with sandwiches often left out of the fridge for extended periods on trays or in patient rooms before being consumed.

Trace back investigation revealed that the minced celery used in sandwiches at both Hospital A and Hospital B was purchased from the same batch from the same local supplier-Processor $A$, and sourced from the Guadalupe region of California. Trace forward investigation revealed that this minced celery product was exclusively supplied to Hospital A and Hospital B, and no other facilities, distributors or stores. Chopped celery from the same batch of product was supplied to a large distribution network exclusive of Hospital A and Hospital B. Inspection of Processor A revealed two concerns. First, pathogen testing was infrequent, with the previous $E$. coli test occurring in January 2020; over a year prior to the present outbreak. Second, while the chlorination step met required standards, it occurred before mincing.

The hypothesized source of this outbreak was minced celery from Processor A. The root cause is hypothesized to be E. coli that persisted after chlorination and was subsequently mixed throughout the product during the mincing process. Temperature 
abuse at Hospital A and Hospital B may have further contributed to propagation of $E$. coli in this product. The outbreak was declared over on May 10, 2021.

As there was no product left when the investigation had reached its conclusion, no product action was taken. Local and federal food safety authorities performed a second, joint inspection of Processor A to make recommendations for more frequent testing for $E$. coli and to conduct a review of the chlorination process. Follow-up was also conducted at Hospital A and Hospital B to propose methods to reduce the likelihood of temperature abuse by using time stamps to record when sandwiches are removed from the fridge.

\section{Discussion}

An outbreak investigation of six cases of E. coli $\mathrm{O} 103$ was conducted in April 2021. The outbreak was associated with consumption of minced celery from a local processor and sourced from California. While this is not the first $E$. coli outbreak reported in celery (11), this is the first to be caused by $E$. coli $\mathrm{O} 103$ and the first to exclusively impact a vulnerable, hospitalized population. This investigation resulted in several recommendations to improve food safety of this food item within the Island Health region.

Evidence from the epidemiological and food safety investigations support minced celery as the source of this outbreak. All six confirmed cases were exposed to the suspect source, and no other product was reported across all six confirmed cases, despite detailed menus for all inpatients. The outlier case, an outpatient who ate a tuna and celery sandwich only during their emergency room visit to Hospital A, added further support to celery as the suspect source. This investigation also revealed strong trace back evidence- - the minced celery served in Hospital A and Hospital B was provided by the same supplier; the investigation also revealed strong trace forward evidencethe supplier provided the minced celery product only to the two hospitals, and nowhere else. Because the contaminated product was no longer available by the time of the investigation, and due to the cleaning procedures at Processor $A$, neither product samples nor environmental samples were available for testing.

Despite the lack of laboratory evidence, the authors believe the strong epidemiological, trace back and trace forward evidence is sufficient to implicate minced celery in this outbreak.

The outbreak highlights the risk of raw vegetables provided to vulnerable populations and draws particular attention to the risk of mincing during processing. While previous work has documented the potential food safety hazards of fresh-cut produce (13), this outbreak serves to document the potential risks posed by mincing, which provides the opportunity for small amounts of bacteria remaining on the surface of a product, even after chlorination, to be spread throughout an entire batch.
Attribution of the mincing step as problematic in this outbreak scenario is further supported as trace forward investigation revealed that more coarsely chopped celery from the same batch was supplied to a wide distribution network, exclusive of Hospital A and Hospital B, with no cases of the outbreak strain of E. coli $\mathrm{O} 103$ associated with this product.

Despite providing food to a population of approximately 800 inpatients each day, identification of only six cases across Hospital A and Hospital B could potentially be explained by a low level of contamination, which may have caused illness only amongst those whose sandwiches were subjected to temperature abuse. Temperature abuse is a known vehicle for pathogen propagation (14-16), and was reported by the hospitals during the investigation follow-up. It is hypothesized that any contamination present after the mincing step in Processor $A$ was further propagated by these reports of temperature abuse, resulting in the illnesses reported. A recommendation was made at the two implicated hospitals to add a time stamp to all sandwiches to mark the time the product was taken out of the fridge, to reduce the risk of temperature abuse moving forward.

There are several limitations to consider in the interpretation of these outbreak data. First, exposure data for celery was not available for the healthy population controls to directly compare with outbreak cases. However, given that $100 \%$ of confirmed cases had exposure to the suspect source, and this was the only common exposure across all six cases, the authors feel confident in the epidemiological evidence for this product. Second, the reporting delay for this outbreak was long, which in turn delayed the outbreak identification and investigation. Reporting delays are influenced by a multitude of factors, but comorbidities among the inpatient and outpatient cases in this outbreak may have delayed consideration of an enteric illness diagnosis and thus the requisition of a stool sample for testing. Third, several cases were missing onset dates as they could not be interviewed. For these individuals, their onset date likely predated their specimen collection date, which would also impact their exposure period. This was taken into account when interpreting the exposure data and analyzing hospital menus. Fourth, there were no food samples available to test for presence of E. coli O103; therefore, there was no laboratory data to definitively confirm the source of this outbreak. However, despite the lack of laboratory confirmation, the authors believe the epidemiological evidence, the trace back data and the trace forward data provided strong support of the suspect source. Lastly, it could not be determined where or how E. coli was introduced, as a further follow-up at the grower in the United States was outside the investigative jurisdiction of this outbreak.

\section{Conclusion}

Raw vegetables, such as celery, are a known source of E. coli contamination and present a risk to vulnerable populations. Mincing during the processing of raw vegetables, and 
temperature abuse prior to consumption, may provide additional layers of risk. This outbreak resulted in several recommendations to reduce the risk of minced celery served in hospitals, including more frequent testing at the processor, a review of the chlorination and mincing process and a review of hospital food services practices to mitigate temperature abuse.

\section{Authors' statement}

CS - Analyzed and interpreted the data and drafted the article AG - Analyzed and interpreted the data and drafted the article SA - Conceptualized the work, interpreted the data and revised the article

$\mathrm{DH}$ - Conceptualized the work, interpreted the data and revised the article

$\mathrm{LH}$ - Analyzed and interpreted the data and revised the article

\section{Competing interests}

None.

\section{Acknowledgements}

None.

\section{Funding}

This work was supported by Island Health.

\section{References}

1. Yang SC, Lin $\mathrm{CH}$, Aljuffali IA, Fang JY. Current pathogenic Escherichia coli foodborne outbreak cases and therapy development. Arch Microbiol 2017;199(6):811-25. DOI PubMed

2. Croxen MA, Law RJ, Scholz R, Keeney KM, Wlodarska $M$, Finlay BB. Recent advances in understanding enteric pathogenic Escherichia coli. Clin Microbiol Rev 2013;26(4):822-80. DOI PubMed

3. Thomas MK, Murray R, Flockhart L, Pintar K, Pollari F, Fazil A, Nesbitt A, Marshall B. Estimates of the burden of foodborne illness in Canada for 30 specified pathogens and unspecified agents, circa 2006. Foodborne Pathog Dis 2013;10(7):639-48. DOl PubMed

4. Tseng M, Sha Q, Rudrik JT, Collins J, Henderson T, Funk JA, Manning SD. Increasing incidence of non-O157 Shiga toxin-producing Escherichia coli (STEC) in Michigan and association with clinical illness. Epidemiol Infect 2016;144(7):1394-405. DOI PubMed

5. Centers for Disease Control and Prevention; List of Selected Multistate Foodborne Outbreak Investigations. Atlanta (GA); CDC; 2021 (accessed 2021-06-21). https://www.cdc.gov/ foodsafety/outbreaks/multistate-outbreaks/outbreaks-list. html
6. Schimmer B, Nygard K, Eriksen HM, Lassen J, Lindstedt BA, Brandal LT, Kapperud G, Aavitsland P. Outbreak of haemolytic uraemic syndrome in Norway caused by stx2positive Escherichia coli O103:H25 traced to cured mutton sausages. BMC Infect Dis 2008;8:41. DOI PubMed

7. Mylius M, Dreesman J, Pulz M, Pallasch G, Beyrer K, Claußen K, Allerberger F, Fruth A, Lang C, Prager R, Flieger A, Schlager S, Kalhöfer D, Mertens E. Shiga toxin-producing Escherichia coli O103:H2 outbreak in Germany after school trip to Austria due to raw cow milk, 2017 - The important role of international collaboration for outbreak investigations. Int J Med Microbiol 2018;308(5):539-44. DOI PubMed

8. Sekse C, O'Sullivan K, Granum PE, Rørvik LM, Wasteson Y, Jørgensen HJ. An outbreak of Escherichia coli O103:H25 bacteriological investigations and genotyping of isolates from food. Int J Food Microbiol 2009;133(3):259-64. DOI PubMed

9. Gaul LK, Farag NH, Shim T, Kingsley MA, Silk BJ, Hyytia-Trees E. Hospital-acquired listeriosis outbreak caused by contaminated diced celery--Texas, 2010. Clin Infect Dis 2013;56(1):20-6. DOI PubMed

10. Erickson MC. Microbial Risks Associated with Cabbage, Carrots, Celery, Onions, and Deli Salads Made with These Produce Items. Compr Rev Food Sci Food Saf 2010;9(6):602-19. DOI PubMed

11. Centres for Disease and Prevention. Multistate Outbreak of Shiga toxin-producing Escherichia coli O157:H7 Infections Linked to Costco Rotisserie Chicken Salad (Final Update). https://www.cdc.gov/ecoli/2015/o157h7-11-15/index.html

12. BC Ministry of Health Services Guidelines and Protocols Advisory Committee. Infectious Diarrhea - Guideline for Ordering Stool Specimens. BC MOH; Mar 16, 2009. https://www2.gov.bc.ca/gov/content/health/practitionerprofessional-resources/bc-guidelines/infectious-diarrhea

13. Francis GA, Gallone A, Nychas GJ, Sofos JN, Colelli G, Amodio ML, Spano G. Factors affecting quality and safety of fresh-cut produce. Crit Rev Food Sci Nutr 2012;52(7):595-610. DOI PubMed

14. Lund BM. Microbiological food safety for vulnerable people. Int J Environ Res Public Health 2015;12(8):10117-32. DOI PubMed

15. Wachtel MR, Charkowski AO. Cross-contamination of lettuce with Escherichia coli O157:H7. J Food Prot 2002;65(3):465-70. DOI PubMed

16. Duffy G, Cummins E, Nally P, O' Brien S, Butler F. A review of quantitative microbial risk assessment in the management of Escherichia coli O157:H7 on beef. Meat Sci 2006;74(1):76-88. DOI PubMed 